\title{
THE DISTRIBUTION OF FOSCHINI'S LOWER BOUND FOR CHANNEL CAPACITY
}

\author{
CHRISTOPHER S. WITHERS, ${ }^{*}$ Industrial Research Limited \\ SARALEES NADARAJAH, ${ }^{* *}$ University of Manchester
}

\begin{abstract}
Foschini gave a lower bound for the channel capacity of an $N$-transmit $M$-receive antenna system in a Raleigh fading environment with independence at both transmitters and receivers. We show that this bound is approximately normal.
\end{abstract}

Keywords: Antenna; capacity; lower bound; transmit receive

2010 Mathematics Subject Classification: Primary 62E17

Secondary 62E20

\section{Introduction}

In wireless communications theory, channel capacity is the maximum number of bits or other information elements that can be handled in a particular channel per unit time. It can also be interpreted as the tightest upper bound on the amount of information that can be reliably transmitted over a channel or the limiting information rate (in units of information per unit time) that can be achieved with arbitrarily small error probability.

Let $X$ and $Y$ denote random variables respectively representing signals that can be transmitted, and signals received, during a block of time over a channel. Let $I(X ; Y)$ denote the mutual information between the random variables $X$ and $Y$. Then the channel capacity can be formally defined as sup $I(X ; Y)$, where the supremum is taken over the space of all distributions for $X$.

Various versions of the channel capacity formula of Foschini (1996) and Foschini and Gans (1998) have been given for different situations with $N$ signals and $M$ receivers. Here, we are interested in the capacity for independent and identically distributed Rayleigh fading channels. These are perhaps some of the simplest fading environments. However, the need to consider channel capacity for such environments has been widespread even in recent years. For example, with respect to performance analysis of adaptive $M$-ary quadrature amplitude modulation systems (see Nechiporenko et al. (2008)); channel coding selection in time-slotted ALOHA packetized multiple-access systems (see Wei et al. (2008)); scheduling algorithms capable of controlling throughput-fairness tradeoff performance (see Lee and Oh (2009)); the use of adaptive source transmission with amplify-and-forward relaying (see Nechiporenko et al. (2009)); the effect of co-channel interference in multiple-input multiple-output systems employing maximal ratio combining (see Rui (2009)); and performance analysis of low density parity check codes with selection diversity combining (see Tan et al. (2010)).

Received 12 July 2010; revision received 21 February 2011.

* Postal address: Applied Mathematics Group, Industrial Research Limited, Lower Hutt, New Zealand.

** Postal address: School of Mathematics, University of Manchester, Manchester M13 9PL, UK.

Email address: mbbsssn2@manchester.ac.uk 
Foschini (1996) and Foschini and Gans (1998) gave the capacity as

$$
C=\log _{2} \operatorname{det}\left(\boldsymbol{I}_{M}+\frac{\rho \boldsymbol{X} \boldsymbol{X}^{\mathrm{H}}}{N}\right)=\sum_{k=1}^{N} \log _{2}\left(1+\frac{\rho\left|\lambda_{k}\right|^{2}}{N}\right)
$$

for no water filling and no correlation either end, where ${ }^{\mathrm{H}}$,denotestheconjugatetranspose, $\left\{\lambda_{k}\right\}$ are the singular values of the $N \times M$ matrix $\boldsymbol{X}=\left(\boldsymbol{X}_{1}, \ldots, \boldsymbol{X}_{N}\right)$, and $\boldsymbol{X}_{1}, \ldots, \boldsymbol{X}_{N}$ are independent complex random $M$-vectors each distributed as a circular symmetric complex normal with mean $\mathbf{0}_{M}$ and covariance the identity matrix $\boldsymbol{I}_{M}$, written $\boldsymbol{X}_{n} \sim \mathcal{C} \mathcal{N}_{M}\left(\mathbf{0}, \boldsymbol{I}_{M}\right)$. Here, $\rho=P_{T} / Q$ is the 'signal-to-noise ratio (SNR)', the ratio of the total transmitted power to the average power of the noise at each receiver.

The lower bound on the capacity given in Foschini and Gans (1998) is

$$
C_{F}=\sum_{k=N-M+1}^{N} \log _{2}\left(1+\frac{\rho \chi_{2 k}^{2}}{N}\right)<C
$$

for $N \geq M$. Now consider the case $M=N$. In this case, the bound was given by Equation (3) of Foschini (1996). Note that $C_{F}$ is the sum of $N$ independent channels with diversity orders from 1 to $N$. This note gives the distribution of this bound. More generally,

$$
C=\log _{2} \operatorname{det}\left(\boldsymbol{I}_{N}+\boldsymbol{X} \boldsymbol{\Gamma} \boldsymbol{X}^{\mathrm{H}}\right)=\log _{2} \operatorname{det}\left(\boldsymbol{I}_{N}+\sum_{k=1}^{N} \Gamma_{k} \boldsymbol{X}_{k} \boldsymbol{X}_{k}^{\mathrm{H}}\right)=\sum_{k=1}^{N} \log _{2}\left(1+\Gamma_{k}\left|\lambda_{k}\right|^{2}\right),
$$

where $\boldsymbol{\Gamma}=\operatorname{diag}\left(\Gamma_{1}, \ldots, \Gamma_{N}\right), \Gamma_{n}=P_{n} / P_{0}, P_{i}$ is the power of the $i$ th interfering signal (the signal that impairs the reception of the wanted signal), and $P_{0}$ is the power of the desired signal. For BLAST (layered space-time) systems, a corresponding formula for band-limited, frequency-selective channels is

$$
C_{F, M F}=\left\langle\sum_{k=1}^{N} \log _{2}\left(1+\Gamma_{k}(f)\right)\right\rangle=\left\langle\sum_{k=1}^{N} \log _{2}\left(1+\boldsymbol{X}_{k}^{\top} \boldsymbol{R}_{k-1}^{-1} \boldsymbol{X}_{k}^{*}\right)\right\rangle
$$

where ${ }^{*}$ ', and ' $T$, denote the complex conjugate and transpose, respectively, and

$$
\langle\cdot\rangle=S \int_{-1 /(2 S)}^{1 /(2 S)} \cdot \mathrm{d} f, \quad \boldsymbol{R}_{k}=\sum_{i=1}^{k} \boldsymbol{X}_{i} \boldsymbol{X}_{i}^{\top}+\mathcal{N},
$$

where $f$ is the frequency, $\Gamma_{k}(f)$, defined by Equation (15) of Ariyavisitakul (2000), is the signalto-interference-plus-noise power density ratio at frequency $f, \mathcal{N}$, defined by Equation (7) of Ariyavisitakul (2000), is an $M \times M$ diagonal matrix with elements determined by the noise power density at frequency $f$ on the $j$ th receiver antenna, $j=1,2, \ldots, M, S$ is the symbol period, and $\boldsymbol{R}_{k}$ is the frequency-domain correlation matrix of the signals on the receiver antennas. He showed that this generalized Foschini lower bound is equal to the true Shannon upper bound $C=\left\langle\operatorname{det}\left(\boldsymbol{R}_{N} \mathcal{N}^{-1}\right)\right\rangle$ when the output SNR of the space-time processing in each layer is represented by the corresponding 'matched filter' bound. So, the bounds are actually achieved.

Using the fact that $\boldsymbol{X} \boldsymbol{X}^{\mathrm{H}}$ of (1.1) is Wishart, Telatar (1999, Equations (7), (10)) showed that (1.1) has mean of the form

$$
\mathrm{E} C=\sum_{j=0}^{M-1} \int_{0}^{\infty} L_{N, M, j}(x) \log _{2}\left(1+\frac{x \rho}{N}\right) \exp (-x) \mathrm{d} x,
$$


where

$$
L_{N, M, j}(x)=\frac{j !}{(j+N-M) !}\left[L_{j}^{N-M}(x)\right]^{2} x^{N-M}
$$

and

$$
L_{k}^{N-M}(x)=\frac{1}{k !} \exp (x) x^{M-N} \frac{\mathrm{d}^{k}}{\mathrm{~d} x^{k}}\left[\exp (-x) x^{N-M+k}\right]
$$

is the associated Laguerre polynomial of order $k$. Note that $L_{N, N, j}(x)=L_{j}(x)^{2}$, where $L_{j}=L_{j}^{0}$ is the Laguerre polynomial of order $j$. For $N=M$, Telatar (1999) showed that E $C$ can be approximated by

$$
N \int_{0}^{4} \log _{2}(1+\rho y) F(y) \mathrm{d} y
$$

for $F(y)=\pi^{-1} \sqrt{y^{-1}-\frac{1}{4}}$. He used $\log$ for $\log _{2}$ throughout. Note that $F(y)$ is the density of the eigenvalues of $\boldsymbol{X} \boldsymbol{X}^{\mathrm{H}}$; see Foschini (1996). From (3.1) below we can write the mean of Foschini's lower bound $C_{F}$ of (1.2) as

$$
\mathrm{E} C_{F}=\sum_{k=1}^{N} \frac{1}{(k-1) !} \int_{0}^{\infty} x^{k-1} \log _{2}\left(1+\frac{2 x \rho}{N}\right) \exp (-x) \mathrm{d} x .
$$

Smith and Shafi (2002) extended Telatar's result for $M=N$ to the variance and found that the Gaussian approximation performs well. Chiani et al. (2003) gave the characteristic function of $C$ in determinant form by obtaining the joint distribution of the eigenvalues of a Wishart matrix, extending the approach of Telatar.

Various approximations for the distribution and density functions of $C$ have been developed. Salo et al. (2004) gave an approximation for the distribution of $C$ for high SNR $\rho$. Approximations for $M \ll N$ and $N \ll M$ are given in Withers (2004), (2006) and Withers and Nadarajah (2010).

There are very few results on the exact distribution of $C$, especially when $M$ and $N$ are of similar magnitude. The one known to us is that due to Smith et al. (2003), where expressions for the distribution and density functions of $C$ are given for $\min (M, N)=1,2,3$. For $\min (M, N)=1$, the given expressions are single finite sums of elementary terms. For $\min (M, N)=2$, the given expressions are double finite sums of terms involving an intractable single integral, the distribution function of generalized inverse Gaussian random variables. For $\min (M, N)=3$, the given expressions are triple finite sums of terms involving an intractable double integral. For $\min (M, N)>3$, expressions can be obtained in principle for the distribution and density functions of $C$. But these are likely to involve intractable triple or higher-order integrals.

So, the expressions of Smith et al. (2003) are of limited use ( $\operatorname{since} \min (M, N)=1,2,3)$ and are clearly complicated. Hence, they cannot be widely used or widely implemented.

The aim of this note is to provide accessible approximations for the distribution of $C$ when $M$ and $N$ are of similar magnitude. The given approximations are not limited for low or high SNR $\rho$. The simplest approximation is to apply the central limit theorem and to assume a normal limit. Cornish-Fisher methods (see Cornish and Fisher (1937) and Fisher and Cornish (1960)) can be used to provide higher-order approximations than those based on the central limit theorem. The central limit theorem approximation can be accurate for sufficiently large $M$ and $N$. If $M$ or $N$ is not large then higher-order corrections can be applied to obtain a desired level of accuracy. Computational tools for the normal distribution are available everywhere, even in many pocket calculators, so our approximations can be widely used and widely implemented. 
In Section 2 we give the distribution of Foschini's lower bound (1.2) and its extension (1.3). We show that the capacity is asymptotically normal. Derivations are given in Section 3. In Section 4 we give a comparison with the distribution of the capacity $C$ of (1.1).

\section{Main results}

Let $C^{\prime \prime}=N^{-1} C_{F} \ln 2$ and $n=N^{2}$. We show that $\kappa_{r}\left(C^{\prime \prime}\right)$, the $r$ th cumulant of $C^{\prime \prime}$, can be expanded in the form

$$
\kappa_{r}\left(C^{\prime \prime}\right) \approx \sum_{i=2 r-2}^{\infty} b_{r, i} N^{-i}
$$

where the coefficients $b_{r, i}$ do not depend on $N$. The use of ' $\approx$ ' indicates an asymptotic expansion that may diverge. To apply Cornish-Fisher methods (see Cornish and Fisher (1937) and Fisher and Cornish (1960)), (2.1) needs to be viewed as an expansion in powers of $n^{-1 / 2}$, whereas standard estimates have expansions in powers of $n^{-1}$; see Withers (1982), (1984). In the latter case, we can use the standard 'derived' Cornish-Fisher expansion given in Withers (1984). In our case, we need the nonstandard 'derived' Cornish-Fisher expansion of Withers (1984). This gives expansions for the distribution of $C^{\prime \prime}$ (and so those of $C_{F}$ ) in powers of $n^{-1 / 2}=N^{-1}$ and the leading cumulant coefficients $b_{r, i}$. For the central limit theorem approximation, we only need the coefficients given by Theorem 2.1.

Theorem 2.1. We have

$$
\begin{aligned}
& b_{1,0}=\left(1+\omega^{-1}\right) \ln (1+\omega)-1, \\
& b_{1,1}=\frac{\omega}{2(1+\omega)} \\
& b_{2,2}=\ln (1+\omega)-\frac{\omega}{1+\omega}=\frac{b_{1,0}}{1+\omega^{-1}},
\end{aligned}
$$

for $\omega=2 \rho$.

The three coefficients of Theorem 2.1 represent the asymptotic mean, first-order bias, and first-order variance of $C^{\prime \prime}$. The asymptotic mean $b_{1,0}$ can be written as

$$
b_{1,0}=\int_{0}^{1} \ln (1+\omega x) \mathrm{d} x \text {. }
$$

This form is given by Equation (5) of Foschini (1996) and Equation (18) of Foschini and Gans (1998), with $\omega=2 \rho$ replaced by $\rho$. This difference is due to their nonstandard scaling of the chi-square random variables. Note that, for large SNR, $b_{2,2} \approx b_{1,0} \approx \ln \omega-1$, so $N \operatorname{var}\left(C_{F}\right) / \mathrm{E} C_{F} \approx 1 / \ln 2$.

Our central limit theorem approximation for $C_{F}$ can now be written as

$$
Y_{N}=b_{2,2}^{-1 / 2}\left(C_{F} \ln 2-N b_{1,0}-b_{1,1}\right) \rightarrow \mathcal{N}(0,1)
$$

as $N \rightarrow \infty$. That is, $Y_{N}$ is approximately a standard real normal random variable with density and distribution

$$
\phi(x)=(2 \pi)^{-1 / 2} \exp \left(-\frac{x^{2}}{2}\right), \quad \Phi(x)=\int_{-\infty}^{x} \phi(y) \mathrm{d} y .
$$




\section{Derivations}

Note that $C_{F}$ of (1.2) can be written as

$$
C_{F}=\sum_{k=1}^{N} \log _{2}\left(1+\frac{\omega G_{k}}{N}\right)
$$

where the $G_{k}=\chi_{2 k}^{2} / 2$ are independent gamma random variables with mean $k$ and density $x^{k-1} \exp (-x) /(k-1)$ !. The cumulants and moments of $G_{k}$ are $\kappa_{r}\left(G_{k}\right)=k(r-1)$ ! and $\mathrm{E} G_{k}^{r}=(k)_{r}$, where $(k)_{r}=\Gamma(k+r) / \Gamma(k)=k(k+1) \cdots(k+r-1)$, using the notation of Abramowitz and Stegun (1964) and Gradshteyn and Ryzhik (1965). To begin with, we study the more general problem

$$
C=\sum_{k=1}^{N} \log _{2}\left(1+\frac{\omega_{k} G_{k}}{N}\right)
$$

where the $\left\{\omega_{k}\right\}$ are assumed to be bounded away from 0 and $\infty$. So, $C=C_{F}$ if $\omega_{k} \equiv \omega$. Set

$$
C^{\prime}=C \ln 2=\sum_{k=1}^{N} X_{k, N}
$$

for $X_{k, N}=\ln \left(1+\omega_{k} G_{k} / N\right)$. By Lyapounov's theorem (see Billingsley (1968, p. 44)), $C$ is asymptotically normal as $N \rightarrow \infty$ if

$$
\sum_{k=1}^{N} \mu_{4}\left(X_{k, n}\right) /\left(\sum_{k=1}^{N} \mu_{2}\left(X_{k, n}\right)\right)^{2} \rightarrow 0
$$

as $N \rightarrow \infty$, where $\mu_{r}(X)=\mathrm{E}(X-\mathrm{E} X)^{r}$. This condition is much weaker than requiring $\left\{\omega_{k}\right\}$ to be bounded away from 0 and $\infty$. We now prove the following.

Lemma 3.1. For $r \geq 1$, the rth cumulant of $C^{\prime}$ is given in terms of the joint cumulants of powers of $G_{k}$ by

$$
\kappa_{r}\left(C^{\prime}\right) \approx \sum_{j=r}^{\infty} N^{-j}(-1)^{j-r} d_{r, j}
$$

for

$$
d_{r, j}=\sum_{k=1}^{N} \omega_{k}^{j} d_{r, j, k}
$$

where

$$
d_{r, j, k}=\sum_{i_{1}+\cdots+i_{r}=j, i_{1} \geq 1, \ldots, i_{r} \geq 1}\left(i_{1} \cdots i_{r}\right)^{-1} \kappa\left(G_{k}^{i_{1}}, \ldots, G_{k}^{i_{r}}\right) .
$$

Proof. Note that

$$
X_{k, n}=\sum_{i=1}^{\infty} w_{i, k, N} G_{k}^{i}
$$

for $w_{i, k, N}=w_{i}\left(\omega_{k} / N\right)^{i}$ and $w_{i}=(-1)^{i-1} / i$. Since the $\left\{X_{k, N}\right\}$ are independent,

$$
\kappa_{r}\left(C^{\prime}\right)=\sum_{k=1}^{N} \kappa_{r}\left(X_{k, N}\right), \quad \kappa_{r}\left(X_{k, N}\right)=\sum_{i_{1}=1}^{\infty} \cdots \sum_{i_{r}=1}^{\infty} w_{i_{1}, k, N} \cdots w_{i_{r}, k, N} \kappa\left(G_{k}^{i_{1}}, \ldots, G_{k}^{i_{r}}\right) .
$$

This completes the proof. 
By (3.2), the $r$ th cumulant of $C^{\prime \prime}=C^{\prime} / N$ has magnitude $N^{2-2 r}=n^{1-r}$ so $C^{\prime \prime}$ has CornishFisher expansions in powers of $n^{-1 / 2}$. For the case $\omega_{k} \equiv \omega, \kappa_{r}\left(C^{\prime \prime}\right)$ can also be expanded in the form (2.1).

Proof of Theorem 2.1. Now take $\omega_{k} \equiv \omega$ so that $C^{\prime \prime}=C^{\prime} / N$ and $C^{\prime}=C_{F} \ln 2$. By Lemma 3.1,

$$
\kappa_{r}\left(C^{\prime}\right)=(-1)^{r} \sum_{j=r}^{\infty}\left(-\frac{\omega}{N}\right)^{j} D_{r, j}
$$

for

$$
D_{r, j}=\sum_{k=1}^{N} d_{r, j, k}
$$

Note that

$$
\sum_{k=1}^{N}(k)_{j}=\frac{(N)_{j+1}}{j+1}
$$

so that

$$
d_{1, j, k}=\frac{(k)_{j}}{j}, \quad D_{1, j}=\frac{(N)_{j+1}}{(j)_{2}} .
$$

By Abramowitz and Stegun (1964, p. 824),

$$
x(x-1) \cdots(x-j+1)=\sum_{m=0}^{j} S_{j}^{m} x^{m}
$$

for $S_{j}^{m}=s(j, m)=(-1)^{m-j}\left|S_{j}^{m}\right|$, the Stirling number of the first kind, tabled there on pages 833-834. So,

$$
(N)_{j}=\sum_{m=0}^{j} S_{j}^{m}(-1)^{m-j} N^{m}=N^{j} \sum_{i=0}^{j} S_{j}^{j-i}(-N)^{-i} .
$$

So, we may take

$$
b_{1, i}=(-1)^{i-1} \sum_{j \geq 1, j \geq i-1} \frac{(-\omega)^{j} S_{j+1}^{j+1-i}}{(j)_{2}} .
$$

Using Abramowitz and Stegun (1964, p. 824), we can show that

$$
\begin{aligned}
S_{j+1}^{j} & =-\frac{(j)_{2}}{2}=-\left(\begin{array}{c}
j+1 \\
2
\end{array}\right), \\
S_{j+1}^{j-1} & =\frac{1}{8}(j-1)_{4}-\frac{1}{6}(j-1)_{3}, \\
S_{j+1}^{j-2} & =-\frac{1}{48}(j-2)_{6}+\frac{1}{12}(j-2)_{5}-\frac{1}{24}(j-2)_{4} .
\end{aligned}
$$

So, (2.2) and (2.3) follow. Also,

$$
\sum_{i=2}^{\infty} b_{2, i} N^{-i} \approx \operatorname{var}\left(C^{\prime \prime}\right) \approx \sum_{j=2}^{\infty} N^{-j-2}(-\omega)^{j} D_{2, j} .
$$


From

$$
d_{2, j, k}=\sum_{i_{1}+i_{2}=j, i_{1} \geq 1, i_{2} \geq 1}\left(i_{1} i_{2}\right)^{-1}\left((k)_{j}-(k)_{i_{1}}(k)_{i_{2}}\right),
$$

we have

$$
\begin{array}{ll}
d_{2,2, k}=(k)_{2}-k^{2}=k, & D_{2,2}=\frac{1}{2}(N)_{2}, \\
d_{2,3, k}=(k)_{3}-k(k)_{2}=2(k)_{2}, & D_{2,3}=\frac{2}{3}(N)_{3}, \\
d_{2,4, k}=2^{-2}\left((k)_{4}-(k)_{2}^{2}\right)+\frac{2}{3}\left((k)_{4}-k(k)_{3}\right)=3(k)_{3}-\frac{1}{2}(k)_{2}, & D_{2,4}=\frac{3}{4}(N)_{4}-\frac{1}{6}(N)_{3},
\end{array}
$$

illustrating that $D_{2, j} \approx(j-1) N^{j} / j$ as $N \rightarrow \infty$. So,

$$
b_{2,2}=\sum_{j=1}^{\infty}(-\omega)^{j}\left(1-\frac{1}{j}\right)
$$

and (2.4) follows. The proof of the theorem is complete.

\section{A comparison with the distribution of the capacity}

Here, we give a comparison with the distribution of the capacity $C$ of (1.1). The asymptotic means of capacity $C$ and $C_{F}$ of (1.1) and its lower bound (1.2) are related as follows:

$$
\frac{b_{1,0}}{\ln 2}=\int_{0}^{1} \log _{2}(1+\omega x) \mathrm{d} x<\log _{2}(1+\rho)
$$

for $\omega=2 \rho$ and

$$
C= \begin{cases}M^{-1} \lim _{N \rightarrow \infty} \mathrm{E} C_{F} & \text { for } M=N \\ M^{-1} \lim _{N \rightarrow \infty} \mathrm{E} C_{F} & \text { for fixed } M \\ N^{-1} \lim _{M \rightarrow \infty} \mathrm{E} C_{F} & \text { for fixed } N\end{cases}
$$

Equation (4.1) follows by Jenson's inequality. The middle equation of (4.2) was given in Foschini and Gans (1998) and follows from the law of large numbers. The last equation of (4.2) follows by a duality; see Withers (2006). The domains of the three equations in (4.2) are exclusive: $M=N, M \ll N$, and $M \gg N$. In Figure 1 we plot both sides of (4.1).

More generally, by Withers and Vaughan (2001) and Withers (2004), (2006), setting $n_{1}=$ $\min (M, N)$ and $n_{2}=\max (M, N)$ for $r \geq 1$ and fixed $n_{1}, C_{0}=C \ln 2$ has the $r$ th cumulant

$$
\kappa_{r}\left(C_{0}\right) \approx \sum_{i=r-1}^{\infty} a_{r, i}\left(n_{1}\right) n_{2}^{-i}
$$

as $n_{2} \rightarrow \infty$ for certain functions $a_{r, i}=a_{r, i}\left(n_{1}\right)$. Those needed for the distribution of $C$ via $Y_{M, N}$ below are given by Example 3.1 of Withers and Nadarajah (2010): $a_{1,0}=n_{1} \ln (1+\rho)$ and $a_{2,1}=n_{1} v_{1}^{2}$ for $v_{1}=\left(1+\rho^{-1}\right)^{-1}$. The first approximation (central limit theorem) for $C$ is

$$
Y_{M, N}=\left(\frac{n_{2}}{a_{2,1}}\right)^{1 / 2}\left(C_{0}-a_{1,0}\right) \rightarrow \mathcal{N}(0,1) \quad \text { as } n_{2} \rightarrow \infty
$$




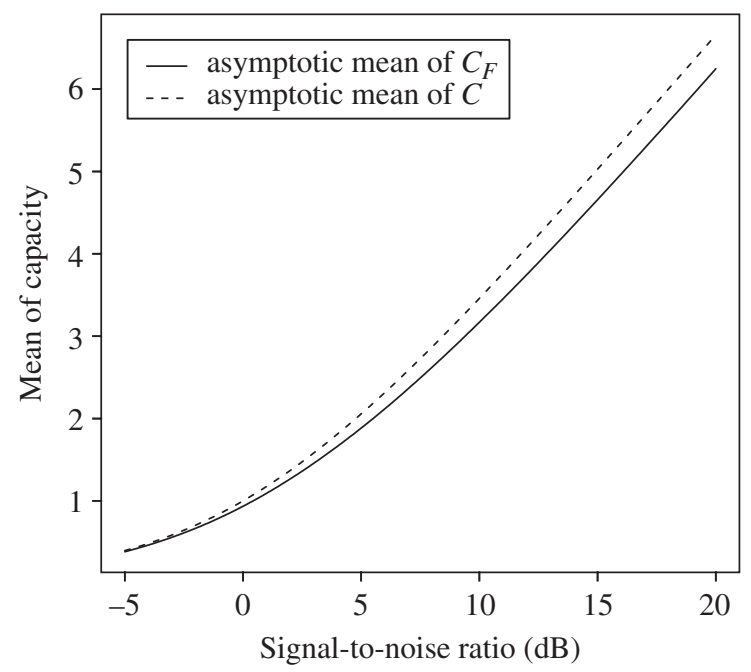

Figure 1: Asymptotic means of $C$ for $M=N$ and $C_{F}$.

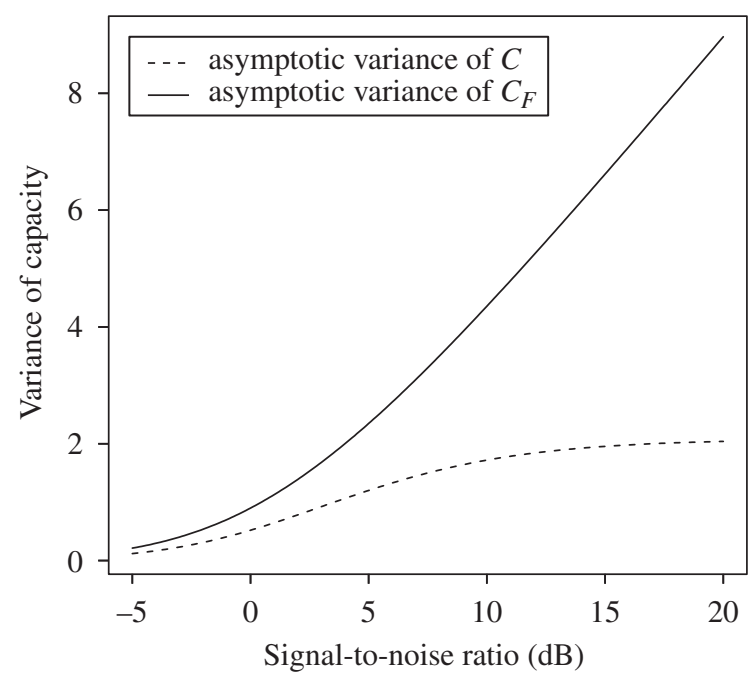

FIGURE 2: Asymptotic variances of $C$ for $M=N$ and $C_{F}$.

Comparing the variances of $C$ and $C_{F}$,

$$
\begin{aligned}
\operatorname{var}(C) & \approx \frac{(\ln 2)^{-2} v_{1}^{2} n_{1}}{n_{2}}=(\ln 2)^{-2} v_{1}^{2} \quad \text { if } M=N, \\
\operatorname{var}\left(C_{F}\right) & \approx(\ln 2)^{-2} b_{2,2} .
\end{aligned}
$$

In Figure 2 we plot these asymptotic variances when $M=N$.

For small $\rho$,

$$
b_{2,2} \approx 2 \rho^{2}-\frac{16}{3} \rho^{3}+\cdots, \quad v_{1}^{2} \approx \rho^{2}-2 \rho^{3}+\cdots,
$$


so that $\operatorname{var}\left(C_{F}\right) \approx 2 \operatorname{var}(C)$ when $M=N$. As $\rho \rightarrow \infty$,

$$
b_{2,2}=\ln \left(\frac{2 \rho}{\mathrm{e}}\right)\left\{1+O\left(\rho^{-1}\right)\right\} \rightarrow \infty, \quad v_{1}^{2}=1+O\left(\rho^{-1}\right) \rightarrow 1,
$$

so that

$$
\operatorname{var}\left(C_{F}\right) \rightarrow \infty,\left.\quad \operatorname{var}(C)\right|_{M=N} \rightarrow \frac{1}{(\ln 2)^{2}}
$$

\section{Acknowledgements}

The authors would like to thank the Editor and the anonymous referee for careful readings and helpful comments, which greatly improved the paper.

\section{References}

Abramowitz, M. and Stegun, I. A. (1964). Handbook of Mathematical Functions with Formulas, Graphs, and Mathematical Tables (Nat. Bureau Standards Appl. Math. Ser. 55). US Government Printing Office, Washington, DC.

Ariyavisitakul, S. L. (2000). Turbo space-time processing to improve wireless channel capacity. IEEE Trans. Commun. 48, 1347-1359.

BillingSley, P. (1968). Convergence of Probability Measures. John Wiley, New York.

Chiani, M., Win, M. Z. And Zanella, A. (2003). On the capacity of spatially correlated MIMO Rayleigh-fading channels. IEEE Trans. Inf. Theory 49, 2363-2371.

Cornish, E. A. AND Fisher, R. A. (1937). Moments and cumulants in the specification of distributions. Rev. Internat. Statist. Inst. 5, 307-320.

Fisher, R. A. AND CoRnish, E. A. (1960). The percentile points of distributions having known cumulants. Technometrics 2, 209-225.

FoschINI, G. J. (1996). Layered space-time architecture for wireless communication in a fading environment when using multi-element antennas. Bell Labs Tech. J. 1, 41-59.

Foschini, G. J. AND Gans, M. J. (1998). On limits of wireless communications in a fading environment when using multiple antennas. Wireless Personal Commun. 6, 311-335.

Gradshteyn, I. S. And Ryzhik, I. M. (1965). Tables of Integrals, Series, and Products, 4th edn. Academic Press, New York.

LEE, M. AND OH, S. K. (2009). A simple scheduling algorithm capable of controlling throughput-fairness tradeoff performance. In IEEE VTS Vehicular Technology Conf. Proc., pp. 1622-1625.

Nechiporenko, T., Phan, K. T., Tellambura, C. and Nguyen, H. H. (2008). Performance analysis of adaptive M-QAM for Rayleigh fading cooperative systems. In Proc. 2008 IEEE Internat. Conf. Commun., pp. 3393-3399.

Nechiporenko, T., Phan, K. T., Tellambura, C. and Nguyen, H. H. (2009). On the capacity of Rayleigh fading cooperative systems under adaptive transmission. IEEE Trans. Wireless Commun. 8, 1626-1631.

RuI, X. (2009). Capacity and SER analysis of MIMO MRC systems in an interference-limited environment. Wireless Personal Commun. 50, 133-142.

Salo, J., Suvikunnas, P., El-Sallabi, H. M. and Vainikainen, P. (2004). Approximate distribution of capacity of Rayleigh fading MIMO channels. Electronics Lett. 40, 741-742.

Smith, P. J. And Shafi, M. (2002). On a Gaussian approximation to the capacity of wireless MIMO systems. In Proc. 2002 IEEE Internat. Conf. Commun., pp. 406-410.

SMith, P. J., GARTH, L. M. AND LoYKa, S. (2003). Exact capacity distributions for MIMO systems with small numbers of antennas. IEEE Commun. Lett. 7, 481-483.

TAN, B. S., Li, K. H. AND TeH, K. C. (2010). Performance analysis of LDPC codes with selection diversity combining over identical and non-identical Rayleigh fading channels. IEEE Commun. Lett. 14, 333-335.

Telatar, E. (1999). Capacity of multi-antenna Gaussian channels. Europ. Trans. Telecommun. 10, 585-595.

Wei, C., FAn, P. AND Ben Letaief, K. B. (2008). On channel coding selection in time-slotted ALOHA packetized multiple-access systems over Rayleigh fading channels. IEEE Trans. Wireless Commun. 7, 1699-1707.

Withers, C. S. (1982). The distribution and quantiles of a function of parameter estimates. Ann. Inst. Statist. Math. 34, 55-68.

Withers, C. S. (1984). Asymptotic expansions for distributions and quantiles with power series cumulants. J. R. Statist. Soc. B 46, 389-396. 
Withers, C. S. (2004). Outage probability for channel capacity for multiple arrays. In Proc. 2004 Austral. Commun. Theory Workshop, pp. 37-41.

Withers, C. S. (2006). Reciprocity for MIMO systems. In Proc. 7th Austral. Commun. Theory Workshop, pp. 175-183.

Withers, C. S. AND NADARAJAH, S. (2010). Channel capacity for MIMO systems with multiple frequencies and delay spread. Techn. Rep., Applied Mathematics Group, Industrial Research Limited.

Withers, C. S. And Vaughan, R. (2001). The distribution and percentiles of channel capacity for multiple arrays. In Proc. 11th Symp. Wireless Commun., pp. 141-152. 